\title{
Pseudoaneurysms Complicating Organ Transplantation: Roles of CT, Duplex Sonography, and Angiography
}

In a retrospective study of proved pseudoaneurysms (PAs) in 15 patients with transplanted organs (11 liver, three kidney, one pancreas), the results of computed tomography (CT), duplex sonography, and angiography were reviewed. Of the 15 cases of PA, eight occurred at the arterial anastomosis and seven were nonanastomotic. Three of the eight anastomotic PAs were caused by infection. Of the seven nonanastomotic PAs, four were caused by percutaneous biopsy, two were caused by infection, and one was of undetermined cause. In nine $(60 \%)$ of the 15 patients the PAs were incidentally detected at imaging studies performed for other reasons. Diagnosis requires a high degree of suspicion. CT was performed in nine cases and duplex sonography in ten. The diagnosis of PA was made with CT in six $(67 \%)$ patients and with duplex sonography in five $(50 \%)$. CT and duplex sonography could not enable diagnosis when the PA was small, when the arterial anastomosis was not included in the field of study, or when enhancement with intravenously administered contrast material was suboptimal. Angiography depicted the PAs in all 15 patients. In three liver transplant recipients with gastrointestinal tract bleeding, the causative PAs were detected only with angiography.

Index terms: Aneurysm, hepatic, 952.458 - Aneurysm, pancreaticoduodenal, 952.458 - Aneurysm, renal, 961.458 - Kidney, transplantation, 81.458 - Liver, transplantation, 761.458 - Pancreas, transplantation, 770.458

Radiology 1988; 169:65-70
V ASCULAR complications are a significant cause of morbidity and mortality following organ transplantation. Arterial complications result in allograft loss in approximately $7 \%$ of liver and $1.5 \%-6 \%$ of kidney transplant recipients $(1-5)$. The most common vascular complication in hepatic and renal allografts is hepatic artery thrombosis and renal artery stenosis, respectively $(2-4,6,7)$. In pancreas transplants, vascular thrombosis and infection are the most frequent complications (8).

A pseudoaneurysm (PA) is an uncommon vascular complication following organ transplantation. The potential for rupture, followed by fatal hemorrhage, makes early diagnosis and treatment of PAs important to patient survival. Generally a complication of vascular reconstruction, PAs usually occur at arterial anastomoses and are often the result of infection. PAs may also occur after percutaneous needle biopsy. Since PAs may be asymptomatic, they are often detected at imaging studies performed for other reasons. Diagnosis of these lesions requires a high degree of suspicion. In this study, we report the computed tomographic (CT), duplex sonographic, and angiographic findings in 15 organ transplant recipients with PAs.

\section{SUBJECTS AND METHODS}

From January 1981 through July 1987. 1,211 liver, 1,162 kidney, and 14 pancreas transplantations were performed at our institution. The current study is a retrospective analysis of the findings in 15 patients who received organ transplants (11 liver, three kidney, one pancreas) and who de-

' From the Departments of Radiology (P.J.T., A.B.Z., J.H.S., A.B., C.R.F., M.L.S., K.M.B.) and Surgery (C.O.E., T.E.S.), University of Pittsburgh School of Medicine, Pittsburgh. From the 1987 RSNA annual meeting. Received March 7, 1988; revision requested April 13; revision received May 11; accepted June 6. Address reprint requests to A.B.Z., Department of Radiology, Presbyterian-University Hospital, DeSoto at O'Hara Sts, Pittsburgh, PA 15213.

c RSNA, 1988

veloped PAs postoperatively (Table 1). Nine of the 15 patients (all liver transplant recipients) were examined with $C T$. Indications for CT in these patients included suspected abdominal abscess $(n=$ 4), graft dysfunction ( $n=1)$, falling hematocrit $(n=1)$, suspected bile leak $(n=$ $1)$, back pain $(n=1)$, and further evaluation of a PA seen at duplex sonography $(n=1)$. All CT scans were obtained on a General Electric 9800 scanner (Milwaukee). Scans were generally obtained at contiguous $1-\mathrm{cm}$ intervals through the entire abdomen following the oral and intravenous administration of contrast material. Contrast material was not given intravenously in patients with a history of allergic reaction or poor renal function.

Ten of the 15 patients (six liver, three kidney, one pancreas recipients) were examined with duplex sonography. Indications for duplex sonography in these patients included graft dysfunction $(n=4)$, assessment of hepatic artery patency $(n=$ $2)$, aspiration of suspected abscess $(n=2)$, exclusion of a diagnosis of abdominal abscess $(n=1)$, and search for a vascular cause of a diminished femoral pulse $(n=$ 1). All duplex examinations were performed on an Acuson 128 (Mountain View, Calif) computed sonography unit.

All 15 patients underwent angiographic evaluation. Findings from only the CT and duplex sonographic examinations that were performed within 2 weeks of angiography were included in this study.

Allograft revascularization followed standard surgical techniques. In seven liver transplants, rearterialization was performed by end-to-end anastomosis between the donor celiac axis and the recipient common hepatic artery. In two liver transplants, revascularization was performed with a donor iliac artery homograft as an interposition arterial conduit between the recipient abdominal aorta and the donor celiac axis. In two liver transplants, the donor aorta with the attached allograft blood supply was used as an arterial conduit from the recipient abdominal aorta. The three renal transplants underwent end-to-side anastomosis of the allograft renal artery to the recipient external iliac artery. The single pancreas transplant was revascularized with the recipient left common iliac artery. 


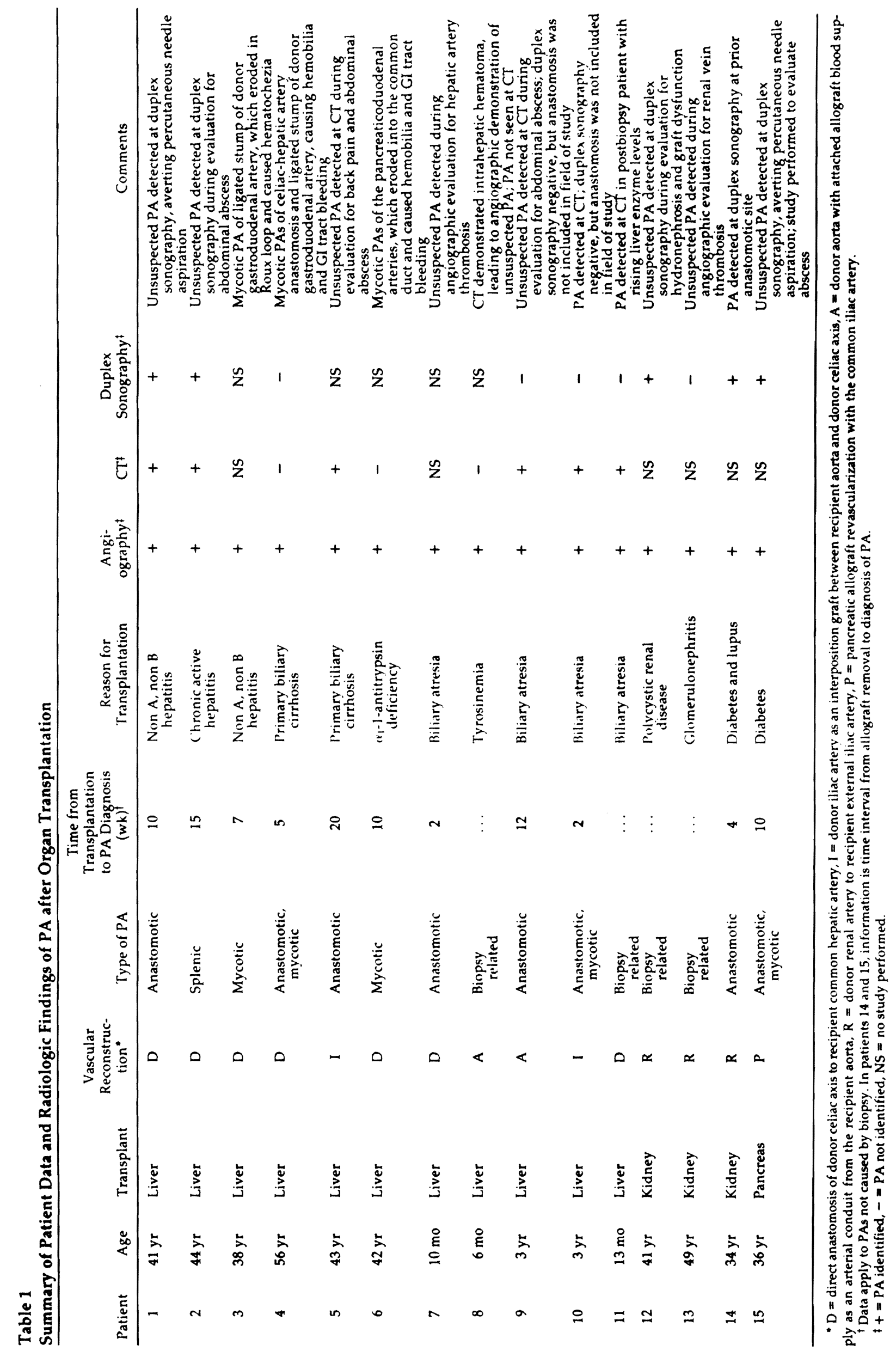




\section{RESULTS}

\section{Liver Transplants}

Eleven of the 15 cases of PA occurred in liver transplant recipients; six were in adults and five were in children (Table 1). The location of the PA in six of the 11 patients was at the arterial anastomosis (patients 1, 4, $5,7,9,10)$, whereas in five it was nonanastomotic (patients 2, 3,6,8, 11 ). The cause of two of the six anastomotic PAs was infection (patients 4, 10). Of the five nonanastomotic PAs, two were caused by infection (patients 3,6 ), two were caused by percutaneous organ biopsy (patients 8 , 11 ), and one was of undetermined cause (patient 2).

Angiography demonstrated the PAs in all 11 liver transplants.
Six $(67 \%)$ of the nine CT scans obtained in the liver transplant recipients demonstrated the PA. Four of the six positive $C T$ scans demonstrated the PA to be at the anastomosis (Fig 1), and two demonstrated it to be nonanastomotic (Fig 2). In three patients $\mathrm{CT}$ failed to demonstrate the $\mathrm{PA}$. In one of the three patients (patient 6 , Fig 3), angiography revealed small aneurysms in the pancreaticoduodenal arteries. In another patient (patient 4), angiography revealed PAs of both the celiac-hepatic artery anastomosis and the ligated stump of the donor gastroduodenal artery. In the third patient (patient 8, Fig 4), angiography revealed a small biopsyrelated PA. In all three cases, intravenously administered contrast material enhancement was either not used (patient 4) or suboptimal because a

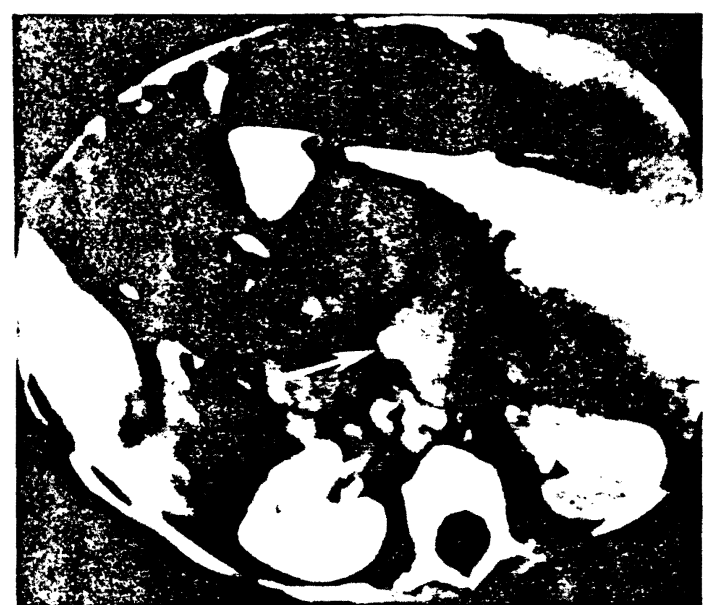

a.

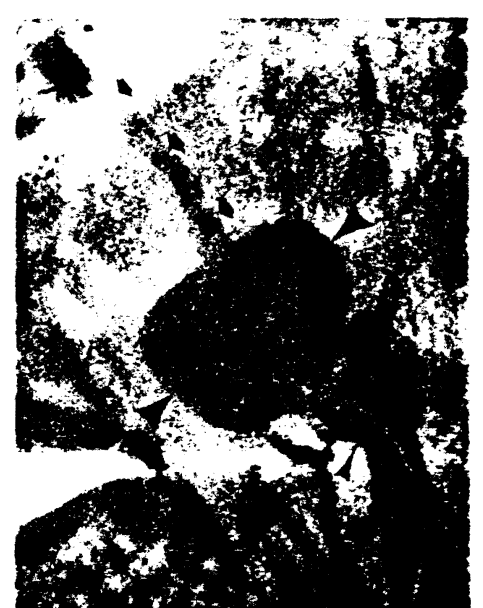

b.

Figure 1. Patient 9. (a) Enhancing mass (arrow) at level of the proximal anastomosis of a donor aortic conduit demonstrated on CT scan. (b) PA (large arrowheads) just distal to the proximal anastomosis (small arrowhead) of a donor aortic conduit demonstrated on right posterior oblique view of selective graft arteriogram. The aortic conduit (arrows) distal to the PA is patent. A dissecting PA was found at surgery.

nonbolus technique was used (patients 6,8$)$. While CT did not demonstrate these PAs, probably because of their small size or poor enhancement, in two patients $C T$ revealed evidence of recent hemorrhage, which was subhepatic in one (patient 4 ) and intrahepatic in the other (patient 8 ,

Fig 4). These findings prompted angiographic evaluation, which documented PAs in both cases.

Results in two (33\%) of the six duplex sonographic examinations performed in the liver transplant recipients (patients 1,2 ) suggested a diagnosis of PA, leading to angiographic evaluation (Fig 5). Of the four cases in which duplex sonography failed to enable diagnosis of the PA, two occurred in patients with a PA at an aortic graft anastomosis (patients 9 , $10)$. In these two cases, the area of the aortic anastomosis was not included in the field of study. In both cases, however, the PA was identified at CT (Fig 1). In the other two patients (patients 4,11 ), sonography demonstrated hypoechoic collections corresponding in size, location, and configuration to the PA diagnosed at angiography. However, since the Doppler cursor was not applied, the sonographic diagnosis of PA was not established in either case.

\section{Kidney Transplants}

Two of the three kidney transplant patients developed biopsy-related PAs (patients 12,13), and the other patient developed an anastomotic PA (patient 14) (Table 1). Two PAs (patients 12,14) were initially detected at duplex sonography and subsequently confirmed with angiography (Fig 6). The other biopsy-related PA (patient 13) was diagnosed inciden-

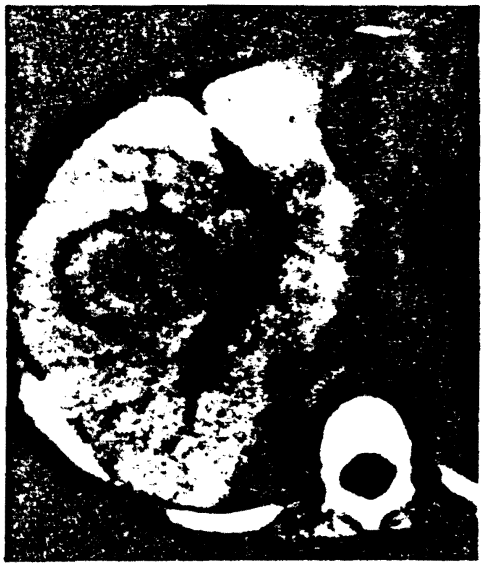

a.

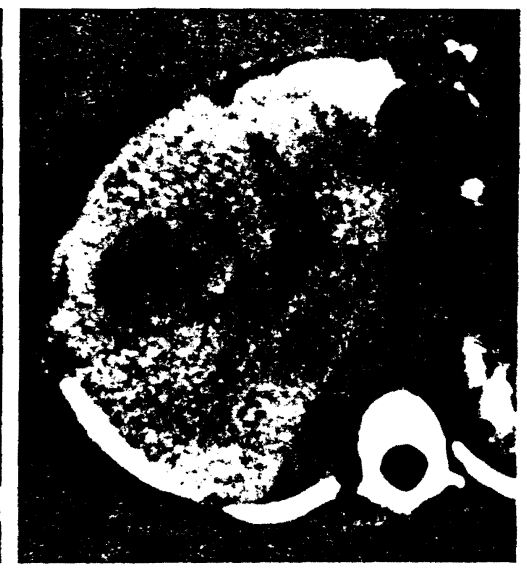

b.

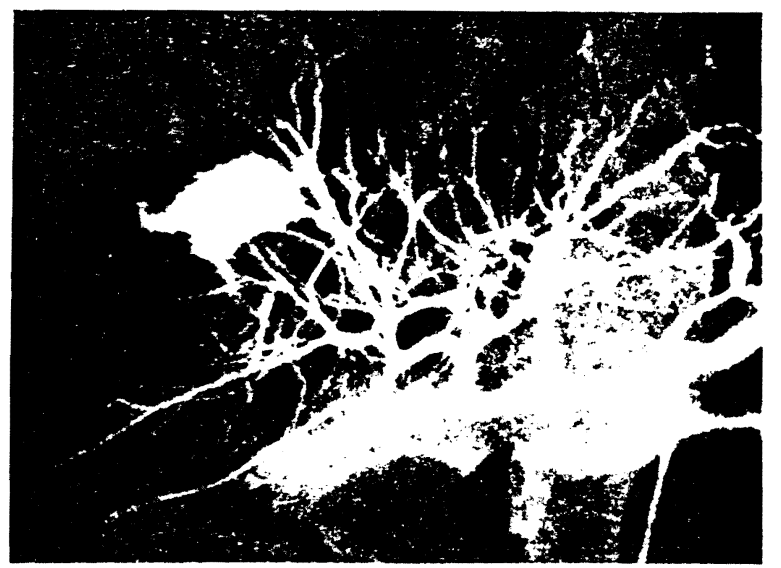

c.

Figure 2. Patient 11. (a) Mixed-attenuation lesion within the right hepatic lobe demonstrated on unenhanced CT scan. (b) After intravenous administration of contrast material, a small enhancing area (arrows) adjacent to the lesion is demonstrated. (c) PA of a branch of the right hepatic artery is demonstrated on celiac arteriogram. 
tally during angiography performed because of decreased allograft function. CT scans were not obtained in these patients.

\section{Pancreas Transplant}

The single pancreas transplant recipient developed a mycotic PA after graft removal at the site of previous arterial anastomosis (patient 15) (Table 1). The PA was initially detected at duplex sonography and subsequently confirmed at angiography. CT was not performed.

\section{DISCUSSION}

In liver transplantation, vascular complications are an important diagnostic consideration in patients with fulminant hepatic failure, bile leak, relapsing bacteremia, gastrointestinal (GI) tract or intraabdominal bleeding, and hemobilia $(1,6)$. The most common and most serious vascular complication is hepatic artery thrombosis. Uncommon vascular complications include hepatic artery stenoses, portal vein stenoses and thromboses, and inferior vena cava thromboses $(1,9)$. Rare but life-threatening vascular complications include anastomotic, mycotic, and biopsy-related PAs.

In renal transplant recipients, vascular complications are a significant cause of graft loss and dysfunction. The reported frequency of vascular complications from renal transplantation varies from $3.5 \%$ to $14 \%$ (2$4,7,10)$. The most common vascular complication is renal artery stenosis, which occurs in $1.5 \%-10 \%$ of patients (2-5). Less common vascular complications include venous thromboses, anastomotic bleeding, arteriovenous fistulas, and PAs. As in liver transplants, PAs in renal allografts may form at the arterial anastomosis $(5,11,12)$, occur after renal biopsy (13), or be due to infection $(5,14,15)$.

The ability to diagnose visceral artery aneurysms with CT and duplex sonography in patients without transplants is well established. CT has been reported useful in the diagnosis of splenic $(16,17)$, hepatic (18$22)$, superior mesenteric (23), and gastroduodenal (24) artery aneurysms and PAs. Sonography has likewise proved useful in the diagnosis of splenic (25), hepatic (26-30), superior mesenteric $(31,32)$, and celiac (33) artery aneurysms and PAs. In renal allografts PAs have traditionally been diagnosed with angiography, but they may also be detected with sonography $(12,13)$ and CT (34). Re- cent reports have stressed the value of duplex sonography in the diagnosis of aneury'sms, since it can demonstrate flow within the aneurysm $(25,27,35)$. In our study, duplex sonography played a diagnostic role in four patients (patients $1,2,12,15$ ) in whom unsuspected PAs were initially detected as incidental findings, prompting angiographic evaluation. In particular, duplex sonography played a crucial role in two of these patients (patients 1, 15) who were referred for sonographically guided needle aspiration of fluid collections Duplex sonography revealed the hypoechoic fluid collections to have mixed arterial and venous flow (Fig 5). A diagnosis of PA was suggested, leading to angiographic documentation. Potentially disastrous needle aspiration was averted.

PAs may be asymptomatic and detected incidentally during imaging evaluation for other reasons, as occurred in nine $(60 \%)$ of the 15 patients (patients 1, 2, 5, 7-9, 12, 13, 15) reported herein. Diagnosis requires a high degree of suspicion. Sites of vascular anastomoses must be scrutinized for evidence of hematoma or abnormal contrast-enhancing areas on CT scans. Intravenously administered contrast material is very helpful, and in three of our cases small PAs may have been missed because contrast material was not used (patient 4) or was used without bolus technique (patients 6,8 ). In two patients (patients 5,10), however, the diagnosis of PA was indicated without the help of contrast enhancement, due to the demonstration of a focal mass adjacent to the aortic anastomosis of iliac interposition grafts. At duplex sonography, any hypoechoic collection in the vicinity of an arterial anastomosis must be evaluated with pulsed Doppler. Detection of arterial flow is an indication for angiography (Fig 5). The importance of evaluating vascular anastomoses is emphasized by the results in four of our cases, in which CT (patients 5, 9; Fig 1) or duplex sonography (patients 1, 15; Fig 5) depicted unsuspected anastomotic PAs, prompting angiographic evaluation. In two patients (patients 9,10), the PA was not detected at duplex sonography, probably because the area of the aortic anastomosis of the conduit grafts was not included in the field of study. These arterial conduit grafts in pediatric liver transplants have proved extremely difficult to image with sonography and constitute a major source of potential diagnostic error.

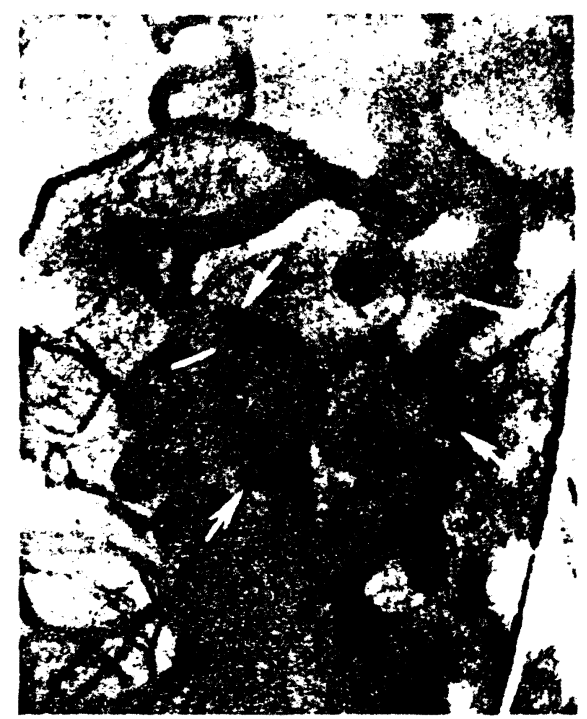

Figure 3. Patient 6. Aneurysms (arrows) in pancreaticoduodenal arterial collaterals demonstrated on superior mesenteric arteriogram. At laparotomy mycotic PAs were found, which had eroded into the common bile duct and caused hemobilia.

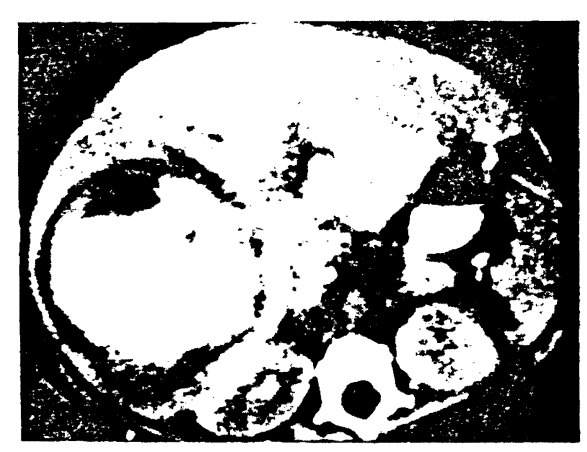

Figure 4. Patient 8. Large hematoma within the right hepatic lobe demonstrated on CT scan. Scan was obtained without bolus technique; the PA was not identified. A small PA from the right hepatic artery was demonstrated on hepatic arteriogram.

Often bowel gas obscures the conduit and anastomosis.

In three of the liver transplant recipients with PAs, GI tract bleeding was the initial symptom. In one patient bleeding was caused by an arterioenteric fistula. A small mycotic PA of the ligated donor gastroduodenal artery eroded into the Roux limb of the jejunum (patient 3 ). In the other two patients GI tract bleeding was due to hemobilia (patients 4,6). Angiography demonstrated the PAs in all three cases. It is noteworthy that in two of these three patients (patients 4,6) a CT scan was obtained, and in one patient (patient 4) duplex sonography was performed before angiography. In none of these three cases did CT or duplex sonography demonstrate the PAs; however, recent subhepatic hemorrhage was 


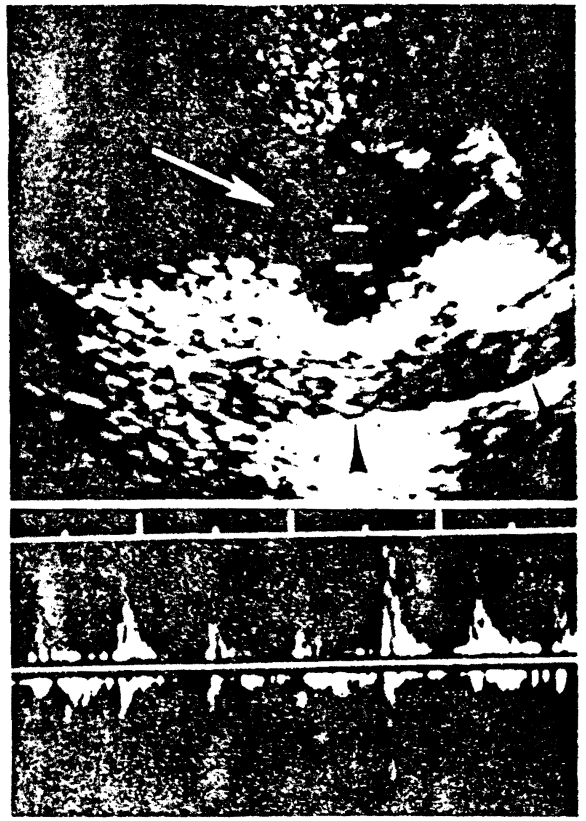

5.

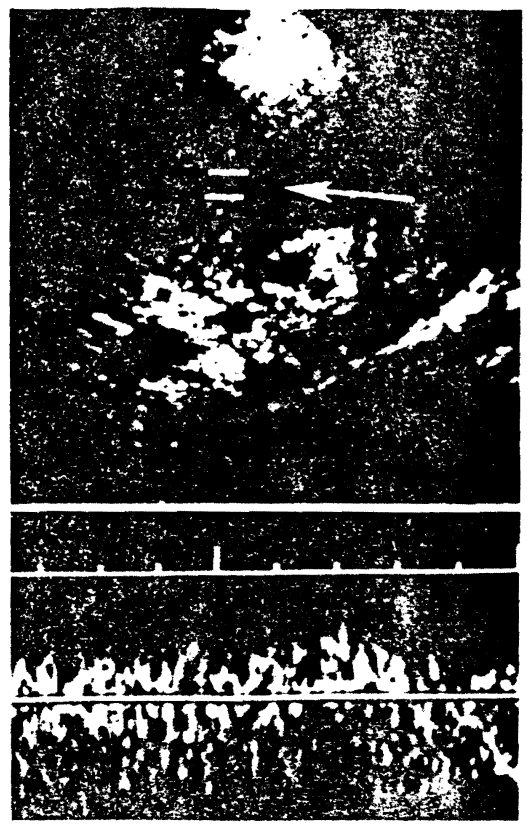

6a.

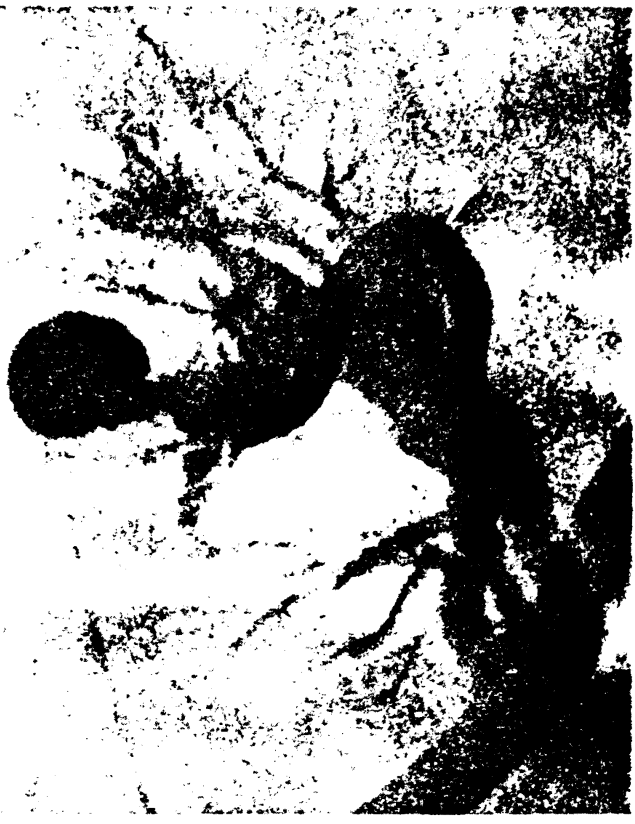

6b.

Figures 5, 6. Patients 1, 12. (5) Patient 1. Hypoechoic mass (arrow) anterior to the aorta (arrowheads) at level of allograft hepatic artery anastomosis demonstrated on parasagittal sonogram. Doppler spectrum demonstrates arterial pulsations within the mass. PA of the hepatic artery anastomosis was found at surgery. (6a) Patient 12. Hypoechoic mass (arrow) within a kidney allograft demonstrated at sonography. Doppler spectrum demonstrates mixed arterial and venous pulsations within the mass. (6b) PA (curved arrow) and arteriovenous fistula with early venous filling (straight arrows) demonstrated on selective external iliac arteriogram. There is enlargement of the feeding artery (arronhead) to the PA and fistula.

identified at CT in one patient (patient 4). In hemodynamically stable patients with hemobilia or with GI tract bleeding and a negative endoscopic evaluation, the initial diagnostic examination should be angiography. Technetium-99m-labeled red blood cell scanning may be helpful to localize a site of GI tract bleeding, thus directing angiographic evaluation. Regardless of the results of radionuclide scanning, angiography should still be performed to exclude a PA as the underlying cause of bleeding. In one of our patients (patient 3 ), results of a Tc-99m-labeled red blood cell study were negative, while angiography demonstrated the causative PA. In all three patients with GI tract bleeding, the PAs were small in caliber (Fig 3), making diagnosis with CT or duplex sonography difficult.

In liver transplant patients who have undergone liver biopsy and have a falling hematocrit (patient 11) or an expanding intrahepatic hematoma (patients 8,11 ), the possibility of a biopsy-related PA with hematoma must be considered. Hematoma and PA are known complications of liver biopsy (36). In patient 8 , an intrahepatic hematoma was seen as a mass of increased attenuation on CT scans (Fig 4). In patient 11 , the small PA was seen after intravenous contrast material enhancement (Fig 2). In both cases $C T$ led to angiographic evaluation and the diagnosis of PA. Since biopsy-related PAs may be small, they may (patient 11, Fig 2) or may not (patient 8 , Fig 4) be seen at CT or duplex sonography. An enlarg. ing intrahepatic hematoma in a patient who has undergone biopsy demands angiographic evaluation.

In kidney transplant patients, duplex sonography is routinely performed to evaluate for complications such as rejection, lymphocele, and hydronephrosis. Sonography is also useful in pancreas transplant recipients for detection of peripancreatic fluid collections and parenchymal abnormalities (37). PAs are rare complications that can be diagnosed with duplex sonography. Any abnormal hypoechoic areas at the arterial anastomosis or within the allograft parenchyma should be evaluated with pulsed Doppler. The possibility of a PA should be considered in all transplant recipients with evidence of infection near the graft (patient 15) or in patients who have undergone biopsy (patients 12,13; Fig 6) and who have deteriorating renal function, massive or prolonged hematuria, or a bruit over the graft. Angiography is necessary to confirm these PAs and assist in preoperative planning. Nonoperative management with embolization may be possible in select cases (13). In our series, embolization was successfully used in one liver (pa- tient 4) and one kidney (patient 12) transplant patient. While CT can demonstrate PAs in renal allografts, this diagnosis requires scanning after intravenous injection of contrast material (34). With a high degree of suspicion by the ultrasonographer and systematic application of the Doppler cursor to suspicious hypoechoic areas, duplex sonography is the screening examination of choice for detecting PA in kidney and pancreas transplant recipients.

In conclusion, because PAs are commonly asymptomatic and detected on imaging studies performed for other reasons, diagnosis requires close scrutiny of arterial anastomoses, beds of infection, or grafts recently sampled for biopsy. Any abnormal hypoechoic or anechoic collection in these areas must be evaluated with Doppler for evidence of arterial flow. In liver transplant recipients with falling hematocrit and no obvious source of bleeding clinically, CT may be helpful in directing angiographic evaluation. In liver transplant recipients with hemobilia or GI tract bleeding and negative results of endoscopy, angiography is the initial diagnostic examination of choice. Preoperative angiography should be performed in all patients.

Acknowledgment: We thank Maggie Zdrodowski and Kimberly Christian for manuscript preparation. 


\section{References}

1. Tzakis AG, Gordon RD, Shaw BW, Iwatsuki S, Starzl TE. Clinical presentation of hepatic artery thrombosis after liver transplantation in the cyclosporin era. Transplantation $1985 ; 40: 667-671$

2. Palleschi J, Novick AC, Braun WE, Magnusson MO. Vascular complications of renal transplantation. Urology 1980; 16:61-67.

3. Rijksen JFWB, Koolen MI, Walaszewski JE, Terpstra JL, Vink M. Vascular complications in 400 consecutive renal allotransplants. J Cardiovasc Surg 1982; 23:91-98

4. Jordan ML, Cook GT, Cardella CJ. Ten years of experience with vascular complications in renal transplantation. J U rol 1982; 128:689-692.

5. Goldman MH, Vineyard GC, Laks H, Wil son RE. A twenty-year survey of arterial complications of renal transplantation. Surg Gynecol Obstet 1975; 141:758-760.

6. Wozney P, Zajko AB, Bron KM, Point S, Starzl TE. Vascular complications after liver transplantation: a 5-year experience. AJR 1986; 147:657-663.

7. Vidne BA, Leapman SB, Butt KM, Kountz SL. Vascular complications in human renal transplantation. Surgery 1976; 79:7781 .

8. Hanto DW, Sutherland DER. Pancreas transplantation: clinical considerations. Radiol Clin North Am 1987; 25:333-343.

9. Zajko AB, Bron KM, Starzl TE, et al. Angiography of liver transplantation patients. Radiology 1985; 157:305-311.

10. Russo VR, Marks C. Renal transplantation: an analysis of operative complications. Am Surg 1976, 42:153-159.

11. Mulderije ED, Berden JHM, Buskens FGM, Raaijmakers PAM, Rosenbusch G. False and true aneurysms of the renal artery after kidney transplantation: a report of two cases. Br J Radiol 1985; 58:896-899

12. Renigers SA, Spigos DG. Pseudoaneu$\mathrm{r} \cdot \mathrm{sm}$ of the arterial anastomosis in a renal transplant. AJR 1978; 131:525-526.

13. Moreau JF, Merland JJ, Descamps JM. Post-biopsy false arterial aneurysm of a transplanted kidney: treatment by transcatheter embolization. J L'rol 1982; 128:116-118.
14. Squifflet JPG, Pirson YD, Dardenne AN, Van Cangh PJ, van Ypersele de Strihou C Alexandre GPJ. Mycotic aneurysm of renal graft artery: diagnosis by ultrasonography. Urology 1983; 22:212-214.

15. Kyriakides C,K, Simmons RL, Najarian JS. Mycotic aneurysms in transplant patients. Arch Surg 1976; 111:472-476.

16. Burke JW, Erickson SJ, Kellum CD, Tegtmeyer CJ, Williamson BRJ, Hansen MF. Pseudoaneurysms complicating pancreatitis: detection by CT. Radiology 1986 ; 161:447-450.

17. Nino-Murcia M, Kurtz A, Brennan RE, Shaw E, Peiken SR, Weiss SM. CT diagnosis of a splenic artery pseudoaneurysm a complication of chronic pancreatitis and pseudocyst formation. J Comput Assist Tomogr 1983; 7:527-529.

18. Shultz S, Druy EM, Friedman AC. Common hepatic artery aneurysm: pseudocyst of the pancreas. AJR 1985; 144:1287-1288.

19. Hügel HE, Oser W, Bodner E. Aneurysm of the proper hepatic artery as a rare source of upper gastrointestinal bleeding Gastrointest Radiol 1986; 11:158-160.

20. Foley WD, Berland LL, Lawson TL, Maddison FE. Computed tomography in the demonstration of hepatic pseudoaneurysm with hemobilia. J Comput Assist Tomogr 1980; 4:863-865.

21. Zachary K, Geier S, Pellecchia C, Irwin G. Jaundice secondary to hepatic artery aneurysm: radiologic appearance and clinical features. Am J Gastroenterol 1986; 81:295298.

22. Kibbler CC, Cohen DL, Cruicshank JK Kushwaha SS, Morgan MY, Dick RD. Use of CAT scanning in the diagnosis and management of hepatic artery aneurysm. Gut 1985; 26:752-756

23. Kopecky KK, Ellis JH, Bies JR, Madura JA. Computed tomographic diagnosis of pancreatic pseudoaneurysm. Comput Radiol $1984 ; 8: 361-364$

24. Borlaza GS, Kuhns LR, Seigel R, Pozderac $R$, Eckhauser $F$. Computed tomographic and angiographic demonstration of gastroduodenal artery pseudoaneurysm in a pancreatic pseudocyst. J Comput Assist Tomogr 1979; 3:612-614.

25. Derchi LE, Biggi E, Cicio GR, Bertoglio C, Neumaier CE. Aneurysms of the splenic artery: noninvasive diagnosis by pulsed Doppler sonography. J Ultrasound Med $1984 ; 3: 41-44$
26. Athey PA, Sax SL, Lamki N, Cadavid G. Sonography in the diagnosis of hepatic artery aneurysm. AJR 1986; 147:725-727.

27. Falkoff GE, Taylor KJW, Morse S. Hepatic artery pseudoaneury'sm: diagnosis with real-time and pulsed Doppler US. Radiology $1986 ; 158.55-56$

28. Rigaux A, Vossen P, Van Baarle A, De Schepper A. Hepatic artery aneurysm: ultrasonic diagnosis. JCU 1986; 14:401403

29. Paolella LP, Scola FH, Cronan JJ. Hepatic artery aneurysm: an ultrasound diagnosis. JCU 1985; 13:360-362.

30. Wolinski AP, Gall WJ, Dubbins PA. Hepatic artery aneurysm following pancreatitis diagnosed by ultrasound. Br J Radiol 1985: 58:768-770.

31. Gooding GAW. Ultrasound of a superior mesenteric artery aneurysm secondary to pancreatitis: a plea for real-time ultrasound of sonolucent masses in pancreatitis. JCU 1981; 9:255-256.

32. Ugolotti U, Miselli A, Mandrioli R, Larini $P$, Ross A. Ultrasound diagnosis of superior mesenteric artery aneurysm: two case reports. JCU 1984; 12:581-584.

33. Herzler GM Silver TM, Graham LM, Stanley JC. Celiac artery aneurysm: ultrasonic diagnosis. JCU 1981; 141-142.

34. Raval B, Balsara V, Kim EE. Computed tomography detection of transplant renal artery pseudoaneurysm. J Comput Tomogr 1985; 9:149-151

35. Chou YH, Tiu CM, Pan HB, Su YG, Chang T. Diagnosis of renal pseudoaneurysms by pulsed Doppler ultrasound. JCU 1985; 13:662-666.

36. Wallace S, Medellin H, Nelson RS. Angiographic changes due to needle biopsy of the liver. Radiology 1972; 105:13-18.

37. Letourneau JG, Maile CW, Sutherland DE Feinberg SB. Ultrasound and computed tomography in the evaluation of pancreat ic transplantation. Radiol Clin North Am 1987 ; 25:345-355. 